\title{
A preliminary review of studies on adaptogens: comparison of their bioactivity in TCM with that of ginseng-like herbs used worldwide
}

\author{
Lian-ying Liao ${ }^{1,2}$, Yi-fan He ${ }^{1,2}$, Li Li ${ }^{1,2}$, Hong Meng ${ }^{1,2}$, Yin-mao Dong ${ }^{1,2}$, Fan Yi ${ }^{1,2^{*}} \mathbb{C}$ and Pei-gen Xiao ${ }^{3}$
}

\begin{abstract}
Modern studies have shown that adaptogens can non-specifically enhance the resistance of human body under a wide range of external stress conditions with a multi-targeted and multi-channel network-like manner, especially by affect the immune-neuro-endocrine system and the hypothalamic-pituitary-adrenal axis. This review article draws the attention to the relationships of adaptogens, tonics from traditional Chinese medicine (TCM) and ginseng-like herbs worldwide, which all have similar plant sources and clinical applications. To clarify the sources and pharmacological mechanisms of these plant-originated adaptogens, which will provide useful information for the utilization of adaptogens to improve the human health. Meanwhile, the TCMs and the world-wide ginseng-like herbs from each region's ethnopharmacology will be beneficial modernization and globalization.
\end{abstract}

Keywords: Adaptogen, Immune-neuro-endocrine system, HPA axis, Tonics, Ginseng-like herbs worldwide

\section{Introduction}

The term of adaptogen was first proposed in 1940 by a scientist from the USSR, namely, N. Lazarev, when he described Schisandra chinensis (Turcz.) Bail. and other herbs with the following definition: plant-originated adaptogens that can non-specifically the enhance human body. According to the primary definition of adaptogens, these substances must meet three criteria: first, adaptogens must to be non-specific and must assist the human body in resisting a wide range of adverse conditions, such as physical, chemical or biological stress. These may include environmental pollution, climate change, radiation, infectious diseases, and interpersonal disharmony. Second, adaptogens must maintain homeostasis in humans, that is, these substances can offset or resist physical disorders caused by external stress. Third,

\footnotetext{
*Correspondence: fantasyee@btbu.edu.cn

${ }^{2}$ Beijing Key Laboratory of Plant Resources Research and Development, Beijing Technology and Business University, No. 11/33, Fucheng Road,

Haidian District, Beijing 100048, People's Republic of China

Full list of author information is available at the end of the article
}

adaptogens must not harm the normal functions of the human body.

Then I.Brekhman, a Soviet scientist, studied ginseng in approximately 1950, he extended the concept of adaptogens as follows: medicines that have similar functions as adaptogens can help the body maintain ideal homeostasis under adverse or stressful conditions.

Again, Brekhman and Dardymov further defined plantoriginated adaptogens in 1969 [1]. According to this definition, plant-originated adaptogens meet four criteria: first, plant-originated adaptogens must reduce the harm caused by stressed states, such as fatigue, infection, and depression; second, plant-originated adaptogens must have positive excitatory effects on the human body; third, in contrast to traditional stimulants, the excitatory effects produced by plant-originated adaptogens must not cause side effects such as insomnia, low protein synthesis, or excessive energy consumption; fourth, plant-originated adaptogens must not harm the human body.

In the 1990s, a group of scientists, comprised of Hildebert Wagner, George Wikman and Alexander Panossian, performed many studies on adaptogens and proposed the 
following definition: adaptogens are natural bioregulators that increase the ability to adapt environmental factors and avoid the damage caused by those factors. In fact, the advantage of adaptogens are they minimized the bodily response to stress, reducing the negative reactions during the alarm phase and eliminating, or at least decreasing, the onset of the exhaustion phase that is part of the socalled general adaptation syndrome [2].

With continuous research for more than half a century, the concept of adaptogens has been continuously modified and perfected. In 1998, the American Food and Drug Administration (FDA) [3] defined an adaptogen as a new kind of metabolic regulator that has been proved to help in environmental adaptation and to prevent external harms. Adaptogen has been generally used as a functional term.

Yance, an American herbal doctor, held a view that adaptogens can improve our ability to recognize, respond, recover, and restore or regenerate. He divided adaptogens into three categories, including primary adaptogens, secondary adaptogens, and adaptogen companions, based on his clinical experience [4]. Primary adaptogens are consistent with the traditional definition of adaptogens and satisfy specific criterias. The criterias contains: abundant scientific research confirmed their adaptogenic characters, guarantee of general resistance and non-specific action in the human body, maintenance or restoration for homeostasis, and adverse- or toxiceffects after prolonged use [5].

Furthermore, some precise scientific experiments demonstrated that adaptogens can enhance the resistance of human body against various external stimuli as non-specific regulators. Adaptogen function mainly by affecting the hypothalamic-pituitary-adrenal (HPA axis) in response to stimulation by external stress. Primary adaptogens can not only maintain or recover homeostasis and allostasis but can also promote anabolic recovery. Primary adaptogens can produce positive stress response and the associated hormone expression. Primary adaptogens strengthen the functioning of each systems, promote optimal response, promote recovery of function, and help regulate energy use by improving the function of neuroendocrine system and enhancing cellular energy transfer, which can make body utilize oxygen, glucose, lipids and proteins very effectively, thus providing us with a steady supply of energy [4].

Another category of adaptogens is secondary adaptogens, which are consistent with most traditional definitions of adaptogens but not all of the criteria of primary adaptogens. Secondary adaptogens cannot influence the HPA axis directly; however, these adaptogens can affect the immune, nervous and endocrine systems. Secondary adaptogens share several common features: first, these adaptogens typically exert influence on the immune, nervous and endocrine systems; second, these adaptogens do not influence the HPA axis, directly; third, these plant-originated adaptogens include fatty acids, sterols and phenols; fourth, these substances can enhance anabolism. While the secondary adaptogens may meet most of the qualifications of primary adaptogens, but they have yet to be studied extensively [5].

Another category is adaptogen companions, which may not satisfy all the traditional standards but can have beneficial effects on the HPA axis and on anabolism to support the functions of adaptogens. Although these kinds of medicinal plants have similar functions as the other two kinds of adaptogens mentioned above, these plants can not formally be called adaptogen. Thus, these plants are classified as adaptogens companions because they can act synergistically with the other two kinds of adaptogens mentioned above, thereby improving the effects of the adaptogens [5].

Currently, studies have confirmed that the following plants are true adaptogens: Panax ginseng C.A.Mey, Schisandra chinensis (Turcz.) Baill., Acanthopanax senticosus (Rupr. et Maxim.) Harms, Rhodiola crenulata (Hook. f. et Thoms) S.H.Fu, and Lepidium meyenii Walp.

According to web of science database, we searched the key word, adaptogen. Based on the analysis of results, we got a conclusion that more and more researches focus on this field over past 10 years and it showed an increasing tendency from 1999 to 2018, which profiles that it is still a worthwhile direction to explore. Furthermore, we also found out that the many published articles concentrated on the direction of pharmacology pharmacy, biochemistry molecular biology, plant sciences and agriculture. It is still worthy to show its historical development and find out the relationships among adaptogen and tonics and ginseng-like herbs worldwide, which can give a hint to further research in plant-originated adaptogen. Summarized the results of previous studies and our own researches focused on tonics from TCM, we launched out our understanding of adaptogen: Herbs which can non-specific and non-toxicity help human body resist the environmental stress to maintain a homeostasis. The mechanism may multi-targets and multi-channel network to the neuroendocrine system. The adaptogens with tonics from TCM and the world-wide ginseng-like herbs have similar phytochemical and pharmacological properties.

\section{The functions of adaptogens}

Adaptogens can affect different tissues and organs, and adjust each of these parts to attain homeostasis. 


\section{Adaptogens and adrenal fatigue}

Adrenals, the glands of stress, mobilize various stress responses to each stress, including physical, biochemical, hormonal, thermal, internal, external, emotional and mental. Stress rather than pathological damage is the primary cause of adrenal fatigue. Excessive stress may be caused by a single strong stimulatory event or by the accumulation of chronic or repeated stress. When the capacity of the adrenals to secrete enough hormones that can make the necessary physiological, and biochemical compensations for that level of stress cannot meet the requirements of continually excessive pressure, adrenal fatigue occurs. The adrenal gland continues to work under adrenal fatigue but cannot maintain normal homeostasis. On the one hand, if the adrenals can deal well with this circumstance and cortisol levels remain adequately elevated to handle the various stresses, over time, the signs and symptoms of metabolic syndrome, such as muscle wastage, hyperglycemia, and suppresses immune or inflammatory responses [6], begin to appear. On the other hand, if the adrenals are not able to meet the demands, adrenal fatigue appears, usually developing more quickly than metabolic syndrome, and which can become so severe as to disable them [7].

The amount of stress hormone produced by the human body increases under external pressure. Adaptogens can increase the effectiveness of adrenal gland secretion, thereby abolishing excess hormone production [8]. Other studies can prove this statement: In 2001, B.T.Gaffney found that the suggested that Panax ginseng inhibits 11-beta hydroxysteroid dehydrogenase one and Eleutherococcus senticosus inhibits catechol-O-methyl transferase, both of which reside in close proximity to stress hormone receptors and catalyze the degradation of stress hormones into inactive compounds [9]. In the absence of stress, adaptogens can accelerate the closure of the adrenal gland. Furthermore, adaptogens can increase cellular energy levels and prevent oxidative damages, leading to the maintenance of normal adrenal function. The following plant-originated adaptogens support adrenal function: Panax quinquefolius L. [10], Withania somnifera [11], Panax ginseng C.A.Mey. [9], Codonopsis pilosula (Franch.)Nannf. [12], Eleutherococcus senticosus (Rupr. \& Maxim.) Maxim. [13], Gynostemma pentaphyllum (Thunb.) Makino, Glycyrrhiza uralensis Fisch.ex DC. [14], Ganoderma Lucidum Karst [15], and Sedum rosea (L.) Scop [16].

\section{Adaptogens and arthritis}

Arthritis is caused by tissue damages and joint diseases, which is typically accompanied by pain and swelling. The most common types of arthritis are osteoarthritis and rheumatoid arthritis. Fibromyalgia maybe an accompanying condition of arthritis; however, it is not considered a form of arthritis because it does not cause inflammation or joint damage.

Adaptogens can reduce arthritis-associated inflammation and pain effectively $[17,18]$. The anti-inflammatory effects of the following plant-originated adaptogens can be used to provide relief rheumatoid arthritis: Withania somnifera [8, 19], Panax ginseng C.A.Mey [20], Gynostemma pentaphyllum (Thunb.) Makino [21], Ganoderma Lucidum Karst [22], Sedum rosea (L.) Scop. [23], and Glycyrhiza uralensis Fisch. ex DC. [24].

\section{Adaptogens and sleep}

Many people suffer from insomnia and other sleeprelated problems. External stress perturbs the normal secretion of circadian cortisol, which is the main cause of sleep-related problems. The secretion of cortisol follows the biological clock and external circadian rhythms. The secretion of cortisol peaks in the morning and then decreases, reaching a minimum value at night. Proper exercise, diet and sleep can help maintain stable cortisol levels in the human body.

Adaptogens help produce cortisol and relieve stress [7, 25]. Studies by Steven Maimes and N. V. Provalova suggest that the following plant-originated adaptogens can act as sleep aids: Panax quniquefolius L. [26], Withania somnifera (L.) Dunal. [27], Schisandra chinensis (Turcz.) Baill. [28], Gynostemma pentaphyllum (Thunb.) Makino [29], and Sedum rosea (L.) Scop [30].

The following plant-originated adaptogens can alleviate the effects of the time difference syndrome, caused by disruption of physiological rhythm of the human body: Panax quniquefolius L., Panax ginseng C.A.Mey, Gynostemma pentaphyllum (Thunb.) Makino, Schisandra chinensis (Turcz.) Baill., and Sedum rosea (L.) [31, 32].

\section{Adaptogens and the neuroendocrine system}

One of the most important functions of adaptogens is their ability to help stabilize the internal environment of the human body by affecting the neuroendocrine system. The chemicals in plant-originated adaptogens enhance the ability to adapt to external environments and avoid damage [33-35]. A unique feature of adaptogens is that these substances affect the neuroendocrine system and the cellular energy system. Aaptogens can increase the rates of oxygen, protein, fat and sugar utilization. In addition to plant-originated adaptogens, other plants may have some of the functions mentioned above, but plantoriginated adaptogens have a broad range of functions and systematically strengthen the stability of the internal environment of the human body $[34,36]$. 


\section{Anti-tumor application of plant-originated adaptogens}

Researchers have found that plant-originated adaptogens have a positive influence on all aspects of the health of animals and humans [37]. Cancers studies have shown that plant-originated adaptogen can reduce the risks of cancers [38].

Plant-originated adaptogens play key roles in antitumor and multifaceted anticancer mechanisms, such as inhibition of cancer cells production, stabilization of the functions of human body, and promotion of cell repair $[39,40]$. The anti-tumor effect of adaptogens is often closely linked to immune mechanisms. In other words, adaptogens can activate macrophages, T-lymphocytes, NK cells and so on to inhibit the growth of tumors and enhance cell-selective apoptosis and intercellular connection [16, 41-43].

The most common chemical anti-tumor medicines currently on the market have side effects such as cytotoxicity and immune suppression. Plant-based immune regulators, for example, plant-originated adaptogens, are generally used as auxiliary treatments to reduce the side effects of these chemical medicines and regain health $[44,45]$. Notably, the application of adaptogens improves the tolerance of humans to drug cytotoxicity [46]. Adaptogens can improve the physical conditions of cancer patients in the following ways: first, as modulators of biological responses, adaptogens can the remodel immune mechanism and non-specifically enhance the resistance of the human body [46]; second, adaptogens can promote the production of marrow, increase the amount of blood cells and reduce infection [47]; third, adaptogens affect the entire body, from cells to organs including the liver, kidney, heart and gastrointestinal tract [7]; fourth, adaptogens can strengthen the lethal effects of chemotherapy and radiotherapy on cancer cells [48]; fifth, adaptogens can inhibit the development of multidrug resistance [49]; sixth, adaptogens can inhibit tumor metastasis and cancer cell aggregation [50]; seven, adaptogens can reduce stress hormone levels during immune dysfunction, which is associated with tumor growth [51].

When cancer cells adapt to chemotherapy, the main consequence is the development of multidrug resistance. The most simple mechanism of the development of multidrug resistance is as follows: anti-tumor drug molecules flow out from cancer cells through membrane channel proteins driven by ATP, especially by adjustment of P-glycoprotein pump (Pgp-pump) and due to the effects of breast cancer resistance protein-1 (BCRP/ABCG-2) and multidrug-resistance-associated protein-1 (MRP-1) from thymic cancer cells [52]. Among plant-originated adaptogens, Panax ginseng C.A.Mey can substantially reduce multidrug resistance by inhibiting Pgp, and it has been shown that Panax ginseng C.A.Mey can prolong the life of the cancer-bearing mice in animal experiments $[24,53]$. In addition, epigallocatechin-gallate (EGCG) in Eurycoma longifolia can inhibit the expression of Bcl-2 to prevent the development multidrug resistance [54].

\section{Exploration of the mechanism of action of adaptogens}

In the case of various stress modes, adaptogens can activate the adjustment of different responses to cope with different forms of stress. Adaptogens are the material basis of the bodily response to the external environment and can act on the immune system and the stress response system, showing in Fig. 1. The non-specific

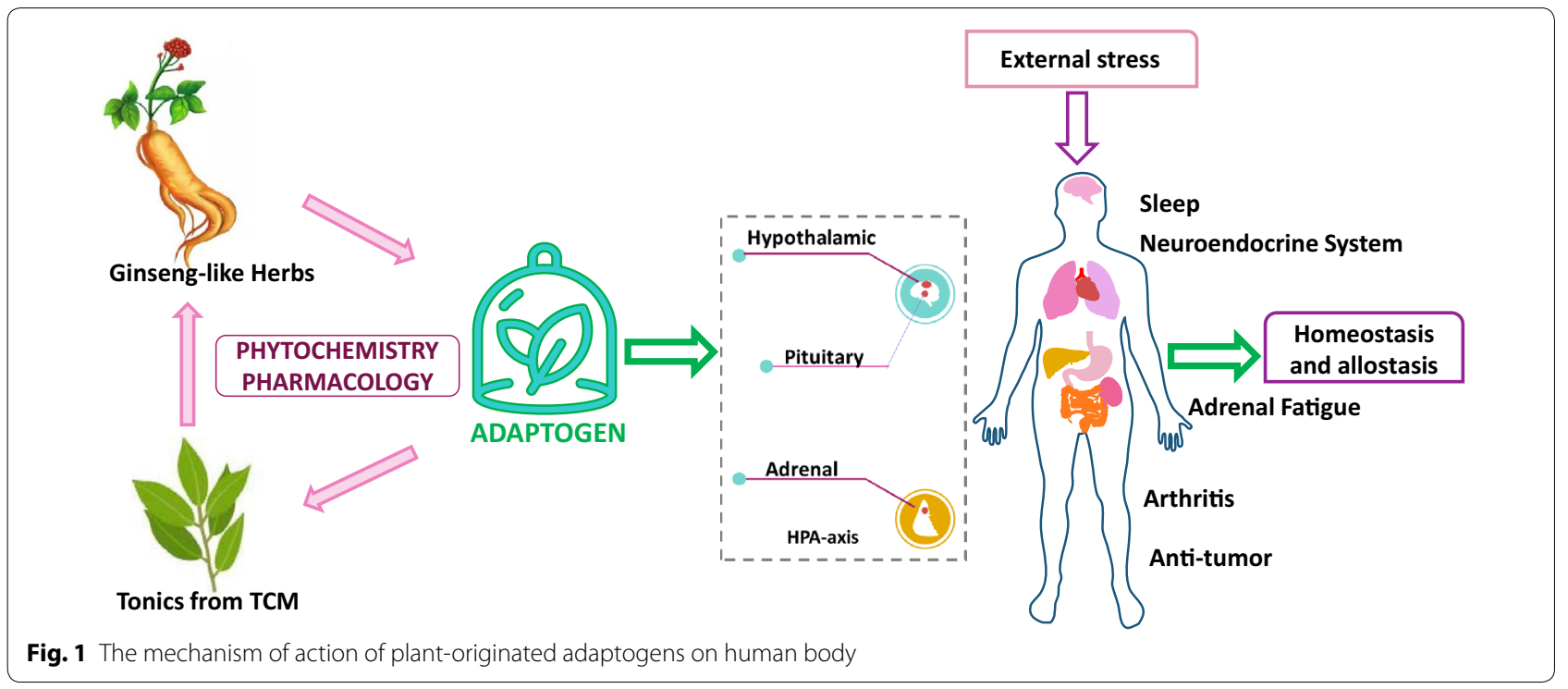


response mode, especially the hormone response mode, occurs when homeostasis is not the driving force. When hormone levels exceed the critical level, the complex neurosecretory reactions may have harmful effects [55, 56]. The human stress response system consists of the central nervous system (CNS), which includes neurons of the hypothalamic paraventricular nucleus, and is associated with to corticotropin releasing hormone $(\mathrm{CRH})$, and arginine vasopressin (AVP), and the adrenalin nucleus as well as its distal ends of the brainstem, the HPA axis and the peripheral nervous system [57]. The central coordination system to respond to external pressure consists of CRH neurons, AVP neurons, catecholamine neurons and other cell tissues, and the HPA axis and sympathetic nervous system (SNS) represent the limbs [58]. CRH and catecholamine neurons interact with each other. The SNS and HPA system interact in terms of functions and systematic anatomy. When responding to the external environment, these systems can interact on different levels, for example, catecholamine can stimulate the HPA axis by releasing of $\mathrm{CRH}$, and the hormone produced by the HPA axis can act on the SNS system [33].

Recent studies have shown that the inhibitory effects and long-term overexpression of endogenous glucocorticoids cause stimulatory effects that are adjusted by SNS under stress. The secretion of CRH and AVP increases, which is stimulated by external pressure, thereby promoting the secretion of cortisol and adrenocorticotropic hormone. Furthermore, angiotensin, cytokines and arachidonic acid metabolites participate in the stress response. SNS provides the human body with a fast response mechanism to external stress. In addition to catecholamine, the sympathetic and parasympathetic nervous systems can also secrete a variety of neuropeptides, ATP and nitric oxide (NO) [59]. The following effects are observed upon adjustment the HPA axis: an increase in and regulation of energy circulation; reduction in the feeling of external pressure; enhancement of resistance; improvement of mental concentration; facilitation of deep sleep period after sleep. These functions are all considered the primary function of adaptogens $[55,56,60]$.

Adaptogens do not increase the levels of cortisol and $\mathrm{NO}$ in the human body under acute physiological loads [6, 31, 61]. Plant-originated adaptogens, for example, Schisandra chinensis (Turcz.) Baill, can prevent and resist stress because these substances can activate the secretion of cortisol and NO in the plasms and saliva, allowing the body to adapt to heavier loads. After the consumption of plant-originated adaptogens, physical exercises do not increase the level of cortisol and NO in the human body; in fact, the levels decrease, comparing to those present prior to physical exercise. Thus, adaptogens can increase the level of messenger substances that activate stress (NO) and suppress stress (cortisol).

Adaptogens can improve the stress response system to respond to high levels of external signals in the normal or abnormal states. We should determine the similarities or differences between adaptogens and known classical metabolic regulators. According to the above description, the main difference may be that adaptogen can stimulate the CNS. It is now possible to obtain additional information at the biochemical level and identify analogues adrenal cortex hormone analogue, catecholamine analogue and so indicating that the active ingredients have similar structures.

\section{Adaptogens and Chinese tonics}

There is much overlap between these medicines and many tonics are internationally recognized as plant-originated adaptogens [2].

In traditional Chinese medicine, it is believed that harmony and balance are indispensable for health and the concepts of yin and yang are used to diagnose and cure disease [62]. Medicines that can supplement deficiency and weakness, correct the pathological bias caused by deficiency in both qi and blood, and treat the syndrome of qi deficiency are called tonics in traditional Chinese medicine (Qì, in the context of Chinese medicine, can be defined as the physiological processes of the body.). According to the differences in the properties, functions and indications of tonics, these medicines can be divided into qi-supplementing medicines, yang-supplementing medicines, blood-supplementing medicines, and yinsupplementing medicines. The common species of tonics and their functions are listed in Table 1.

Tonics have wide range of applications in traditional Chinese medicine and can be used under the conditions of low body resistance and weak constitution or when the human body is finding it difficult to fight severe diseases, this function of tonics is similar to that of plant-originated adaptogens [78]. Tonics typically are adaptogens, but not always. They are similar in the following aspects: First, the clinical application and mechanisms of action of these substances are similar. Based on their current applications, tonics and plant-originated adaptogens can be described as substances that regulate human bodily functions in order to attain homeostasis. The primary effects exhibited by these substances are regulation of the immune system, improving the disorders of the nervous system, anti-fatigue effects and overall nourishment. In terms of specific mechanisms, both medicines can affect the HPA axis in the immune-neuro-endocrine system, thereby achieving the above pharmacological effects [79]. Second, the plants of origin of these substances are similar [2]. Chinese herbs considered to be both adaptogens 


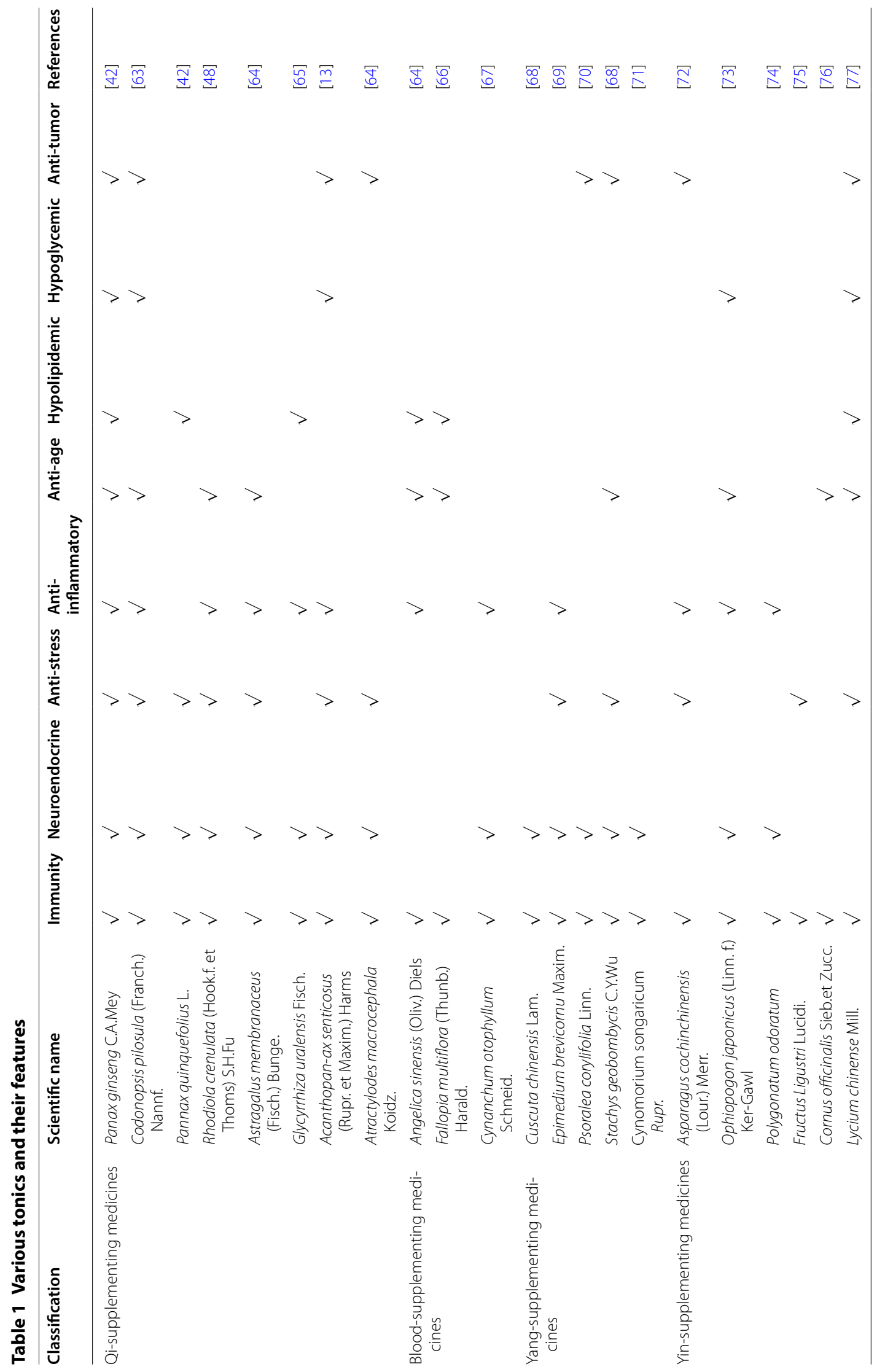


and tonics include the following: Panax ginseng C.A.Mey, Panax quniquefolius L., Panax notoginseng (Burkill) F.H.Chen, Eleutherococcus senticosus (Rupr. \& Maxim.) Maxim, Sedum rosea (L.) Scop., and Schisandra chinensis (Turcz.) Baill. According to the terminology used in traditional Chinese medicine, the mechanism of action of plant-originated adaptogens is to achieve equilibrium in both yin and yang, showing great vitality [80].

Based on the chemical composition, common tonics can be divided into following several categories in Table 2.

According to Panossian's conclusion, the main active chemical components can be divided into the following two categories. The first category includes terpenoids with four-ring skeletons that are similar to cortisol: sitoindosides (Withania somnifera), cucurbitacin-R-saponin (Bryonia dioica). The second category includes aromatic compounds with structures similar to that of catecholamine: (a) lignans: eleuteroside E (Acanthopanax senticosus (Rupr. et Maxim) Harms), schisandrin b (Schisandra chinensis (Turcz.)); (b) phenylpropane derivatives: syringin (Acanthopanax senticosus (Rupr. et Maxim) Harms), cinnamyl glycoside (Rhodiola crenulata (Hook.f. et Thoms) S.H.Fu); (c) phenylethane derivatives: salidroside.

Tonics have the following functions: enriching and activating blood (promoting the secretion of erythropoietin and inducing the production of stimulatory factors, for example, macrophage colonies, to activate blood), regulating cellular and humoral immunity, and affecting cytokine activity. These functions are similar with those of the plant-originated adaptogens mentioned above. Plant-originated adaptogens are generally considered to be the "elite of herbs" [5], and in Chinese medicine, tonics are considered the highest grade of medicine [81]. In traditional Chinese medicine theory, tonics are also considered to be top grades in traditional Chinese medicine. It is helpful to expand the range of plant-originated adaptogen species and to have an in-depth analysis about the mechanism of tonic traditional Chinese medicine.

\section{Adaptogens and ginseng species worldwide}

Many regions, ethnic groups and countries, they have their own medical histories and habits. Continuous development and transfer of knowledge through generations leads to the formation of unique medicinal systems such as traditional Chinese medicine and Indian ayurvedic medicine. Coincidentally, in different regions and medical systems, there are several medicinal plants that are considered to be national treasures or that are called ginseng locally. There is much overlap between plant-originated adaptogens and ginseng species worldwide. Furthermore, most ginseng species worldwide are internationally recognized as plant-originated adaptogens. Both ginseng-like herbs worldwide and plant-originated adaptogens have very similar clinical applications. Ginseng species are widely used by local communities world-wide because these plants can enhance the resistance of the human body and can have various beneficial effects, such as anti-fatigue, anti-ageing, anti-stress, antianxiety, anti-inflammatory, and anti-depression. Furthermore, ginseng species may improve the circulatory system and immune system [34], which correlates precisely to the function of adaptogens.

Most medicinal plants called ginseng belong to Araliaceous, but there are also other medicinal plants that belong to other families. For example, Withania somnifera, which belongs to Slanaceae, is called Indian ginseng; this plant has nourishing and strengthening effects and can delay ageing [47]. Panax japonicus, which belongs to Solanaceae is called Japanese ginseng; this plants have tonifying, strengthening and anti-fatigue effects [82]. Eurycoma longifolia, which belongs to Simaroubaceae is called Malaysian ginseng; this plant can be used as a postpartum tonic or aphrodisiac [83]. Lepidium meyerii, which belongs to Brassicaceae, is called Peruvian ginseng; this plant can be used for natural nutrient, can enhance fertility effectively, and has anti-fatigue effects. The common ginsengs species from different countries and their functions are listed in The results of modern pharmacological studies show that these medicinal plants called ginseng have effects on the neuroendocrine and immune systems, which is similar to the mechanism of action of plant-originated adaptogens, the elite of herbs. However, there have been few studies on the chemical compositions, mechanisms of action, traditional curative effects and similarities of these types of medicinal plants. Comparisons with ginseng species from around the world are helpful for widening the spectrum of plant-originated adaptogen species and for in-depth analysis of the mechanism of action of ginseng species worldwide. The functions and the main functional ingredients of common ginseng-like herbs worldwide were showed in Table 3.

\section{Current difficulties and prospects of adaptogens}

Plant-originated adaptogens have been demonstrated to regulate stress-related changes, at least in animal experiments. However, even after more than 40-years of herbal research, there are very few drugs that have been successfully introduced as adaptogens in modern medicine. Most of these kinds of plant-based medicines are considered to be plant-originated adaptogens and the remaining few are immune enhancers, anabolic agents and antioxidants, which are the same as plant-originated adaptogens. Therefore, there are many difficulties associated 
Table 2 Based on the chemical composition, common tonics can be divided into following several categories

\begin{tabular}{|c|c|}
\hline Category & Specific tonics \\
\hline \multirow[t]{15}{*}{ Saponin } & Panax ginseng C.A.Mey. \\
\hline & Panax japonicus C.A.Mey. \\
\hline & Codonopsis pilosula (Franch.) Nannf. \\
\hline & $\begin{array}{l}\text { Codonopsis lanceolata (Siebold \& Zucc.) Benth. \& } \\
\text { Hook.f. ex Trautv. }\end{array}$ \\
\hline & Glycyrrhiza uralensis Fisch. ex DC. \\
\hline & Eleutherococcus senticosus (Rupr. \& Maxim.) Maxim. \\
\hline & Ophiopogon japonicus (Thunb.) Ker Gawl. \\
\hline & Asparagus cochinchinensis (Lour.) Merr. \\
\hline & Cornus officinalis Siebold \& Zucc. \\
\hline & Trigonella foenum-graecum L. \\
\hline & Ipomoea batatas (L.) Lam. \\
\hline & Ziziphus jujuba Mill. \\
\hline & Broussonetia papyrifera (L.) L’Hér. ex Vent. \\
\hline & Cynomorium songaricum Rupr. \\
\hline & Ligustrum lucidum W.T.Aiton \\
\hline \multirow[t]{7}{*}{ Coumarin } & Psoralea corylifolia L. \\
\hline & $\begin{array}{l}\text { Clinopodium megalanthum (Diels) C.Y.Wu \& S.J.Hsuan } \\
\text { ex H.W.Li }\end{array}$ \\
\hline & Lycium chinense Mill. \\
\hline & Glycyrrhiza uralensis Fisch. ex DC. \\
\hline & Eleutherococcus senticosus (Rupr. \& Maxim.) Maxim. \\
\hline & Panax ginseng C.A.Mey. \\
\hline & Astragalus membranaceus Fisch. ex Bunge \\
\hline \multirow[t]{10}{*}{ Flavonoids } & Epimedium brevicornu Maxim. \\
\hline & Astragalus membranaceus Fisch. ex Bunge \\
\hline & Panax ginseng C.A.Mey. \\
\hline & Eucommia ulmoides Oliver \\
\hline & Psoralea corylifolia L. \\
\hline & Glycyrrhiza uralensis Fisch. ex DC. \\
\hline & Cuscuta chinensis Lam. \\
\hline & Trigonella foenum-graecum L. \\
\hline & Taxillus sutchuenensis (Lecomte) Danser \\
\hline & Dendrobium nobile Lindl. \\
\hline \multirow[t]{11}{*}{ Alkaloids } & Trigonella foenum-graecum L. \\
\hline & Epimedium brevicornu Maxim. \\
\hline & Cistanche deserticola Ma \\
\hline & Polygonatum odoratum (Mill.) Druce \\
\hline & Phlomis umbrosa Turczaninow \\
\hline & Ilex cornuta Lindl. \& Paxton \\
\hline & Allium tuberosum Rottler ex Spreng. \\
\hline & Cuscuta chinensis Lam. \\
\hline & Dendrobium nobile Lindl. \\
\hline & Aconitum carmichaeli Debeaux \\
\hline & Lilium brownii var.viridulum Baker \\
\hline \multirow[t]{3}{*}{ Volatile oils } & Atractylodes macrocephala Koidz. \\
\hline & Alpinia oxyphylla Miq. \\
\hline & Angelica sinensis (Oliv.) Diels \\
\hline
\end{tabular}

Table 2 (continued)

\begin{tabular}{ll}
\hline Category & Specific tonics \\
\hline & Clinopodium megalanthum (Diels) C.Y. Wu \& Hsuan \\
& ex H.W. \\
& Psoralea corylifolia L. \\
& Epimedium brevicornu Maxim. \\
& Trigonella foenum-graecum L. \\
& Rehmannia glutinosa (Gaertn.) DC. \\
& Stachys geobombycis C.Y.Wu \\
& Asparagus cochinchinensis (Lour.) Merr. \\
& Glycyrrhiza uralensis Fisch. ex DC. \\
& Panax ginseng C.A.Mey \\
& Trigonella foenum-graecum L. \\
& Lycium chinense Mill. \\
& Astragalus membranaceus Fisch. ex Bunge \\
& Eleutherococcus senticosus (Rupr. \& Maxim.) Maxim. \\
& Polygonatum sibiricum Redouté \\
& Panax ginseng C.A.Mey. \\
& Fallopia multiflora (Thunb.) Haraldson \\
Polysacchariden & Cynanchum otophyllum C.K.Schneid. \\
& Rehmannia glutinosa (Gaertn.) DC. \\
& Epimedium brevicornu Maxim. \\
& Eucommia ulmoides Oliver \\
Other &
\end{tabular}

with judging whether a plant is a plant-originated adaptogen or not [94].

Adaptogens are stress response modifiers that non-specifically increase resistance to various stressors, thereby promoting adaptation and survival. Adapting to environmental challenges are multistep processes that involve diverse mechanisms and interactions. Multiple molecular networks are involved that coordinate both intracellular and extracellular stress signaling. The metabolic regulation of homeostasis by adaptogens at the cellular and systems levels is associated with multiple targets [95]. To date, the main problem in the research of mechanism of adaptogens, is the lack of suitable stress-response animal models. The stress responses system can be divided into three parts: stressor, stress response and stress performance [96]. The stressor is the object that induces physical strain; therefore, a stressor may be biological (infection), physical (external force, extreme environment), chemical (medicine, ethanol), or psychological (sadness, argument). When an organism is exposed to a stressor, the neuroendocrine system of the body changes. It is easy to study stress performances because these effects are visible [97].

Therefore, stress is known to lead to high blood pressure, myocardial ischemia, depression and even cancer. Briefly, stress performance is the manifestation of the final effect of the stress response on the target organ. 


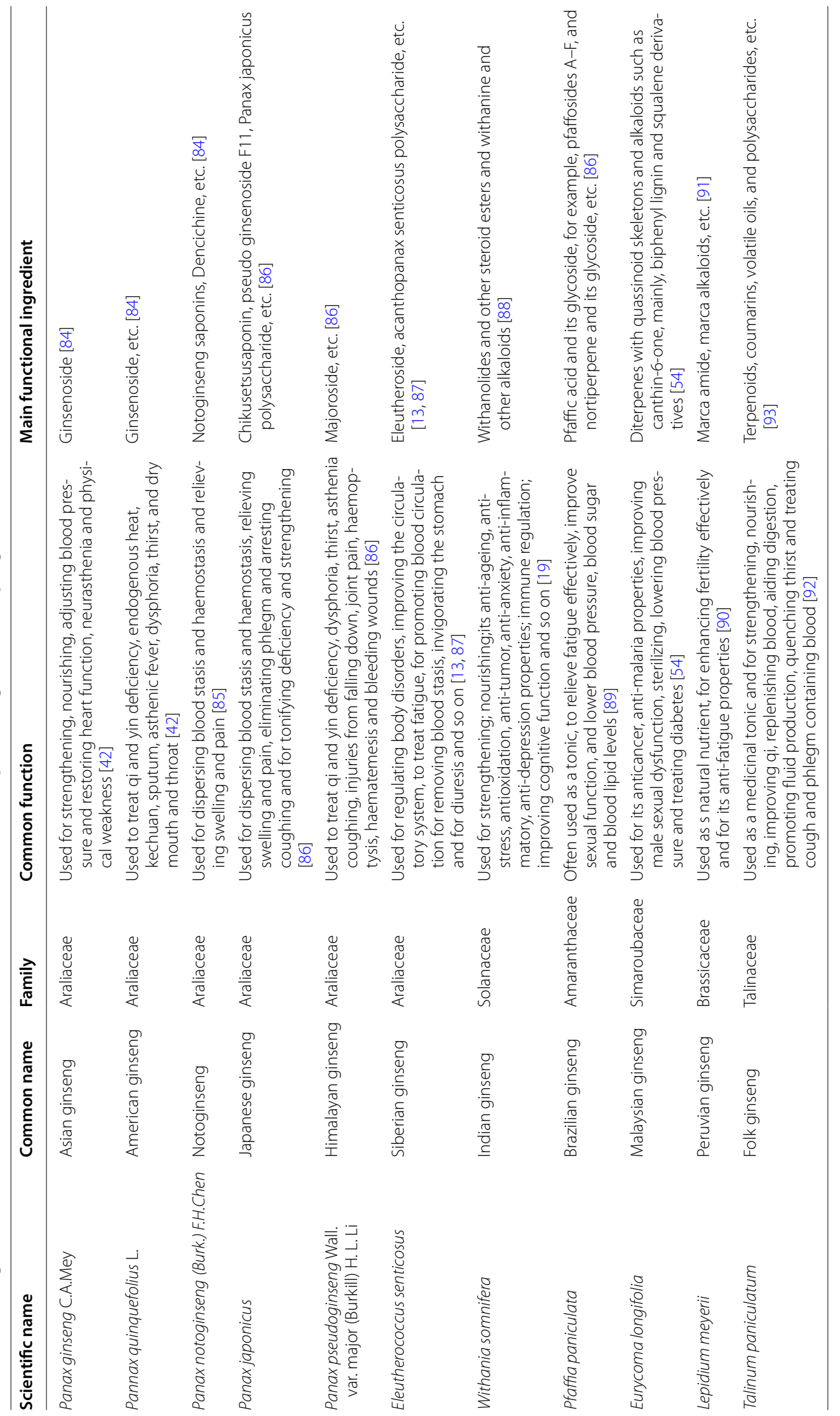


However, it remains challenging to study various steps of this stress response [98]. First, this difficulty is associated with the stressor because it is difficult to quantify external stressors, especially psychological stressors, for example, sadness. In addition, we know little about the interactions between stressors and the body. The specific response of mechanisms induced by stressors affect broad-spectrum stress response. Second, this difficulty is associated with the stress response. In the terms of development, hormones, neurotransmitters, neuro-regulators and cytokines, which are involved in the response have been identified, but the specific functions of these proteins divisions remain unknown. The identity of the first substance to regulate the stress response remains unclear, and it has not been determined whether there are different mechanisms for different stressors. There are no definitive results regarding the specific mechanisms and pathway changes of that lead to conversion of the status from unsuitable to suitable. As a result, it is impossible to differentiate and characterize the different stages of stress response (including adaptation), making the response difficult to quantify [97]. Therefore, scientists use "prevention of stress manifestation" as a guideline to evaluate the anti-stress properties of a drug [99]. Third, the difficulty is associated with stress expression. According to the strength and severity of the stressor, different changes are observed. Genetic or other external factors (species, day-night cycles, gender, age and physiological state of tissues or organs) also affect the extent of overall stress expression. The difficulties mentioned above will appear in the process of the mechanism of action of adaptogens; therefore, it is necessary to further discuss the mechanisms, targets, similarities and differences associated with the pharmacological functions.

To date, various studies and practical applications have shown that plant-originated adaptogens are a kind of elite herbal medicine, playing an important role in human health and helping the human body resist various stress factors. However, the clinical application of plant-originated adaptogens and their use in health care products remains in the preliminary stage. Categorization of plant-originated adaptogens, clarification of their pharmacological functions, and the determination of the similarities and differences between adaptogens, tonics and ginseng species worldwide, will help in effective utilization of plant-originated adaptogens, and provide a new way to guarantee human health.

\section{Authors' contributions}

Conceptualization, P-GX and FY; Methodology, LL, HM, and Y-MD; Writingoriginal draft preparation, L-YY; Writing—review and editing, Y-FH. All authors read and approved the final manuscript.

\begin{abstract}
Author details
${ }^{1}$ Key Laboratory of Cosmetics, China National Light Industry, Beijing Technology and Business University, No. 11/33, Fucheng Road, Haidian District, Beijing 100048, People's Republic of China. ${ }^{2}$ Beijing Key Laboratory of Plant Resources Research and Development, Beijing Technology and Business University, No. 11/33, Fucheng Road, Haidian District, Beijing 100048, People's Republic of China. ${ }^{3}$ Institute of Medicinal Plant Development, Chinese Academy of Medical Sciences, Peking Union Medical College, Beijing, 151 Malianwa North Road, Haidian District, Beijing 100193, People's Republic of China.
\end{abstract}

\section{Acknowledgements}

We are very grateful to Pr. Yong PENG (Institute of Medicinal Plant Development, Chinese Academy of Medical Sciences, Peking Union Medical College, Beijing) for his valuable suggestions.

\section{Competing interests}

The authors declare that they have no competing interests.

\section{Availability of data and materials}

The datasets used or analyzed during the current study are available from the corresponding author on reasonable request.

\section{Consent for publication}

I, the corresponding author give my consent for my article to be publish in Chinese Medicine.

\section{Ethics approval and consent to participate}

Not applicable.

\section{Funding}

This study was supported by the Beijing Technology and Business University Youth Scholars Fund (PXM2018_014213_000033) and the Key Laboratory of Cosmetics, China National Light Industry, Beijing Technology and Business University Innovation Fund (KLC-2017-YB2).

\section{Publisher's Note}

Springer Nature remains neutral with regard to jurisdictional claims in published maps and institutional affiliations.

Received: 23 Auqust 2018 Accepted: 12 November 2018 Published online: 16 November 2018

References

1. Brekhman II, Dardymov IV. New substances of plant origin which increase nonspecific resistance. Ann Rev Pharmacol Toxicol. 1969;9(1):419-30.

2. Mendes FR. Tonic, fortifier and aphrodisiac: adaptogens in the Brazilian folk medicine. Revista Brasileira de Farmacognosia. 2011;21(4):754-63.

3. Winslow LC, Kroll DJ. Herbs as medicines. Arch Intern Med. 1998;158(20):2192-9.

4. Yance DR, Tabachnik B. Breakthrough solutions in herbal medicine adaptogenic formulas: the way to vitality. Townsend Lett. 2007;282:86-90.

5. Yance DR. Adaptogens in medical herbalism: elite herbs and natural compounds for mastering stress, aging, and chronic disease. New York: Simon and Schuster; 2013. p. 103-7.

6. Panossian A, Wikman G. Evidence-based efficacy of adaptogens in fatigue, and molecular mechanisms related to their stress-protective activity. Curr Clin Pharmacol. 2009;4(3):198-219.

7. Winston D, Maimes S. Adaptogens: herbs for strength, stamina, and stress relief. Rochester: Inner Traditions/Bear \& Co; 2007. p. 48-53.

8. Bhatia N, Jaggi AS, Singh N, Anand P, Dhawan R. Adaptogenic potential of curcumin in experimental chronic stress and chronic unpredictable stress-induced memory deficits and alterations in functional homeostasis. J Nat Med. 2011;65(3-4):532-43.

9. Gaffney B, Hügel H, Rich P. Panax ginseng and Eleutherococcus senticosus may exaggerate an already existing biphasic response to stress via inhibition of enzymes which limit the binding of stress hormones to their receptors. Med Hypotheses. 2001;56(5):567-72. 
10. Awang D. The anti-stress potential of North American ginseng (Panax quinquefolius L.). J Herbs Spices Med Plants. 1998;6(2):87-91.

11. Sengupta P, Agarwal A, Pogrebetskaya M, Roychoudhury S, Durairajanayagam D, Henkel R. Role of Withania somnifera (Ashwagandha) in the management of male infertility. Reprod Biomed Online. 2017;36:311-26.

12. Liu K, Wang Z, Rong P. Puerarin decoction with cassia twig combined with auricular acupoint plaster and acupuncture in the treatment of 38 tension headache patients. Int J Clin Acupuncture. 2017;26(1):871

13. Huang L, Zhao H, Huang B, Zheng C, Peng W, Qin L. Acanthopanax senticosus: review of botany, chemistry and pharmacology. Int J Pharm Sci. 2011;66(2):83-97.

14. Wang J, Gao W, Zhang L, Huang L. Establishment and quality assessment of tissue cultures in Glycyrrhiza uralensis Fisch. Appl Biochem Biotechnol. 2013;169(2):588-94.

15. Lu H, Kyo E, Uesaka T, Katoh O, Watanabe H. A water-soluble extract from cultured medium of Ganoderma lucidum (Rei-shi) mycelia suppresses azoxymethane-induction of colon cancers in male F344 rats. Oncol Rep. 2003;10(2):375-9.

16. Chen SP, Huang Liu R, Lu TM, Wei JCC, Wu TC, Tsai WY, et al. Complementary usage of Rhodiola crenulata (L.) in chronic obstructive pulmonary disease patients: the effects on cytokines and T cells. Phytother Res. 2015;29(4):518-25.

17. Bhattacharya A, Ghosal S, Bhattacharya S. Anti-oxidant effect of Withania somnifera glycowithanolides in chronic footshock stress-induced perturbations of oxidative free radical scavenging enzymes and lipid peroxidation in rat frontal cortex and striatum. J Ethnopharmacol. 2001;74(1):1-6.

18. Kropotov A, Kolodnyak O, Koldaev V. Effects of Siberian ginseng extract and ipriflavone on the development of glucocorticoid-induced osteoporosis. Bull Exp Biol Med. 2002;133(3):252-4.

19. Singh N, Bhalla M, de Jager P, Gilca M. An overview on ashwagandha: a Rasayana (Rejuvenator) of ayurveda. Afr J Tradit Complement Altern Med. 2011:8:5S.

20. Choi YS, Kang EH, Lee EY, Gong HS, Kang HS, Shin K, et al. Joint-protective effects of compound $\mathrm{K}$, a major ginsenoside metabolite, in rheumatoid arthritis: in vitro evidence. Rheumatol Int. 2013;33(8):1981-90.

21. Razmovski-Naumovski V, Huang TH-W, Tran VH, Li GQ, Duke CC, Roufogalis BD. Chemistry and pharmacology of Gynostemma pentaphyllum. Phytochem Rev. 2005:4(2-3):197-219.

22. Ho YW, Yeung JS, Chiu PK, Tang WM, Lin ZB, Man RY, et al. Ganoderma lucidum polysaccharide peptide reduced the production of proinflammatory cytokines in activated rheumatoid synovial fibroblast. Mol Cell Biochem. 2007:301(1-2):173-9.

23. Perfumi M, Mattioli L. Adaptogenic and central nervous system effects of single doses of $3 \%$ rosavin and $1 \%$ salidroside Rhodiola rosea $\mathrm{L}$. extract in mice. Phytother Res. 2007;21(1):37-43.

24. Zhu L, Wei H, Wu Y, Yang S, Xiao L, Zhang J, et al. Licorice isoliquiritigenin suppresses RANKL-induced osteoclastogenesis in vitro and prevents inflammatory bone loss in vivo. Int J Biochem Cell Biol. 2012;44(7):1139-52

25. Provalova N, Skurikhin E, Pershina O, Suslov N, Minakova MY, Dygai $A$, et al. Mechanisms underling the effects of adaptogens on erythropoiesis during paradoxical sleep deprivation. Bull Exp Biol Med. 2002:133(5):428-32.

26. Chanana P, Kumar A. GABA-BZD receptor modulating mechanism of Panax quinquefolius against 72-h sleep deprivation induced anxiety like behavior: possible roles of oxidative stress, mitochondrial dysfunction and neuroinflammation. Front Neurosci. 2016;10:84.

27. Deshpande A, Irani N, Balakrishnan R. Study protocol and rationale for a prospective, randomized, double-blind, placebo-controlled study to evaluate the effects of Ashwagandha (Withania somnifera) extract on nonrestorative sleep. Medicine. 2018;97:26

28. Zhang C, Zhao X, Mao X, Liu A, Liu Z, Li X, et al. Pharmacological evaluation of sedative and hypnotic effects of schizandrin through the modification of pentobarbital-induced sleep behaviors in mice. Eur J Pharmacol. 2014;744:157-63.

29. Takemoto T, Arihara S, Nakajima T, Okuhira M. Studies on the constituents of gynostemma-pentaphyllum makino. 1. structures of gypenoside-I-XIV. yakugaku zasshi. J Pharm Soc Japan. 1983;103(2):173-85.

30. Darbinyan V, Aslanyan G, Amroyan E, Gabrielyan E, Malmström C, Panossian A. Clinical trial of Rhodiola rosea L. extract SHR-5 in the treatment of mild to moderate depression. Nordic J Psychiatry. 2007;61 (5):343-8.
31. Panossian A, Wikman G, Kaur P, Asea A. Adaptogens exert a stressprotective effect by modulation of expression of molecular chaperones. Phytomedicine. 2009;16(6-7):617-22.

32. Provino R. The role of adaptogens in stress management. Aus J Med Herbalism. 2010;22(2):41.

33. Panossian A, Wagner H. Stimulating effect of adaptogens: an overview with particular reference to their efficacy following single dose administration. Phytother Res. 2010;19(10):819-38.

34. Panossian A, Wikman G. Effects of adaptogens on the central nervous system and the molecular mechanisms associated with their stress-protective activity. Pharmaceuticals. 2010;3(1):188-224.

35. Carlini E. Plants and the central nervous system. Pharmacol Biochem Behav. 2003;75(3):501-12.

36. Mendes FR, Carlini EA. Brazilian plants as possible adaptogens: an ethnopharmacological survey of books edited in Brazil. J Ethnopharmacol. 2007;109(3):493-500

37. de Oliveira DR, Leitão SG. Fortifier, tonic, and rejuvenating plants and the adaptogen concept. Berlin: Introduction to Ethnobiology: Springer; 2016. p. 151-61.

38. Arushanyan E. Systemic and cellular mechanisms of anti-tumor activity of plant adaptogens. Vopr Onkol. 2009:55(1):15-23.

39. Wiegant F, Surinova S, Ytsma E, Langelaar-Makkinje M, Wikman G, Post J. Plant adaptogens increase lifespan and stress resistance in C. elegans. Biogerontology. 2009;10(1):27-42.

40. laremenko K. Adaptogens as means of the prevention of malignant tumors. Vopr Onkol. 1989;35(8):912-9.

41. Winters M. Ancient medicine, modern use: Withania somnifera and its potential role in integrative oncology. Altern Med Rev. 2006;11:4.

42. Patel S, Rauf A. Adaptogenic herb ginseng (Panax) as medical food: status quo and future prospects. Biomed Pharmacother. 2017;85:120-7.

43. Kaur P, Makanjuola VO, Arora R, Singh B, Arora S. Immunopotentiating significance of conventionally used plant adaptogens as modulators in biochemical and molecular signalling pathways in cell mediated processes. Biomed Pharmacother. 2017;95:1815-29.

44. Loo WT, Jin L, Chow LW, Cheung MN, Wang M. Rhodiola algida improves chemotherapy-induced oral mucositis in breast cancer patients. Expert Opin Invest Drugs. 2010;19(sup1):S91-100

45. Barton DL, Soori GS, Bauer BA, Sloan JA, Johnson PA, Figueras C, et al. Pilot study of Panax quinquefolius (American ginseng) to improve cancerrelated fatigue: a randomized, double-blind, dose-finding evaluation: NCCTG trial N03CA. Supportive Care Cancer. 2010;18(2):179.

46. Barton DL, Liu H, Dakhil SR, Linquist B, Sloan JA, Nichols CR, et al. Wisconsin Ginseng (Panax quinquefolius) to improve cancer-related fatigue: a randomized, double-blind trial, N07C2. J Natl Cancer Instit. 2013;105(16):1230-8

47. Mishra L-C, Singh BB, Dagenais S. Scientific basis for the therapeutic use of Withania somnifera (ashwagandha): a review. Altern Med Rev. 2000;5(4):334-46.

48. Chiang H-M, Chen H-C, Wu C-S, Wu P-Y, Wen KC. Rhodiola plants: chemistry and biological activity. J Food Drug Anal. 2015;23(3):359-69.

49. Treasure J, editor. Herbal medicine and cancer: an introductory overview. Seminars in oncology nursing. New York: Elsevier; 2005

50. Tavakoli J, Miar S, Zadehzare MM, Akbari H. Evaluation of effectiveness of herbal medication in cancer care: a review study. Iranian J Cancer Prev. 2012;5(3):144

51. Meissner HO, Mscisz A, ReichBilinska H, Mrozikiewicz P, BobkiewiczKozlowska T, Kedzia B, et al. Hormone-Balancing effect of Pre-Gelatinized organic maca (Lepidium peruvianum Chacon): (III) clinical responses of early-postmenopausal women to Maca in double blind, randomized, placebo-controlled, crossover configuration, outpatient study. Int J Biomed Sci. 2006;2(4):375-94.

52. Chai S, To KK, Lin G. Circumvention of multi-drug resistance of cancer cells by Chinese herbal medicines. Chin Med. 2010;5(1):26.

53. Yang Z, Wang J-R, Niu T, Gao S, Yin T, You M, et al. Inhibition of P-glycoprotein leads to improved oral bioavailability of compound $K$, an anticancer metabolite of red ginseng extract produced by gut microflora. Drug Metabol Disposit. 2012;40(8):1538-44.

54. Rehman SU, Choe K, Yoo HH. Review on a traditional herbal medicine, Eurycoma longifolia Jack (Tongkat Ali): its traditional uses, chemistry, evidence-based pharmacology and toxicology. Molecules. 2016;21(3):331. 
55. Panossian A, Oganessian A, Ambartsumian M, Gabrielian E, Wagner H, Wikman G. Effects of heavy physical exercise and adaptogens on nitric oxide content in human saliva. Phytomedicine. 1999;6(1):17-26.

56. Panossian A, Wikman G, Wagner H. Plant adaptogens III. Earlier and more recent aspects and concepts on their mode of action. Phytomedicine. 1999;6(4):287-300.

57. Chrousos GP, Gold PW. The concepts of stress and stress system disorders: overview of physical and behavioral homeostasis. JAMA. 1992;267(9):1244-52.

58. Chrousos GP. Stress and disorders of the stress system. Nat Rev Endocrinol. 2009;5(7):374

59. Wardle J, Sarris J. Clinical naturopathy: an evidence-based guide to practice. New York: Elsevier Health Sciences; 2014.

60. Panossian A, Hambardzumyan M, Hovhanissyan A, Wikman G. The adaptogens Rhodiola and Schizandra modify the response to immobilization stress in rabbits by suppressing the increase of phosphorylated stressactivated protein kinase, nitric oxide and cortisol. Drug Target Insights. 2007;2:117739280700200011.

61. Darbinyan V, Kteyan A, Panossian A, Gabrielian E, Wikman G, Wagner H. Rhodiola rosea in stress induced fatigue-a double blind cross-over study of a standardized extract SHR-5 with a repeated low-dose regimen on the mental performance of healthy physicians during night duty. Phytomedicine. 2000;7(5):365-71.

62. Unschuld PU. Huang Di Nei Jing Su Wen Nature, Knowledge, Imagery in an Ancient Chinese Medical Text: With an Appendix: The Doctrine of the Five Periods and Six Qi in the Huang Di Nei Jing Su Wen. California: Univ of California Press; 2003.

63. Gao S-M, Liu J-S, Wang M, Cao T-T, Qi Y-D, Zhang B-G, et al. Traditional uses, phytochemistry, pharmacology and toxicology of Codonopsis: a review. J Ethnopharmacol. 2018;219:50-70.

64. Xie J-H, Jin M-L, Morris GA, Zha X-Q, Chen H-Q, Yi Y, et al. Advances on bioactive polysaccharides from medicinal plants. Crit Rev Food Sci Nutr. 2016;56(sup1):S60-84.

65. Asl MN, Hosseinzadeh HJP, Derivatives TEoNP. Review of pharmacological effects of Glycyrrhiza sp. and its bioactive compounds. Phytother Res. 2008;22(6):709-24.

66. Lin L, Ni B, Lin H, Zhang M, Li X, Yin X, et al. Traditional usages, botany, phytochemistry, pharmacology and toxicology of Polygonum multiflorum Thunb.: a review. J Ethnopharmacol. 2015;159:158-83.

67. Parker S, May B, Zhang C, Zhang AL, Lu C, Xue CC. A pharmacological review of bioactive constituents of Paeonia lactiflora Pallas and Paeonia veitchii Lynch. Phytother Res. 2016:30(9):1445-73.

68. Donnapee S, Li J, Yang X, A-h Ge, Donkor PO, X-m Gao, et al. Cuscuta chinensis Lam.: a systematic review on ethnopharmacology, phytochemistry and pharmacology of an important traditional herbal medicine. J Ethnopharmacol. 2014;157:292-308

69. Chen X-J, Tang Z-H, Li X-W, Xie C-X, Lu J-J, Wang Y-T. Chemical constituents, quality control, and bioactivity of Epimedii Folium (Yinyanghuo). Am J Chin Med. 2015;43(05):783-834.

70. Chopra B, Dhingra AK, Dhar KLJF. Psoralea corylifolia L. (Buguchi)_folklore to modern evidence. Fitoterapia. 2013:90:44-56.

71. Cui Z, Guo Z, Miao J, Wang Z, Li Q, Chai X, et al. The genus Cynomorium in China: an ethnopharmacological and phytochemical review. J Ethnopharmacol. 2013;147(1):1-15.

72. Negi J, Singh $P$, Joshi G, Rawat M, Bisht V. Chemical constituents of Asparagus. Pharmacognosy Rev. 2010;4(8):215.

73. Chen $\mathrm{M}-\mathrm{H}$, Chen X-J, Wang M, Lin L-G, Wang Y-TJJ. Ophiopogon japonicus - a phytochemical, ethnomedicinal and pharmacological review. J Ethnopharmacol. 2016;181:193-213.

74. Zhao P, Zhao C, Li X, Gao Q, Huang L, Xiao P, et al. The genus Polygonatum: a review of ethnopharmacology, phytochemistry and pharmacology. J Ethnopharmacol. 2017;214:274-91.

75. Gao L, Li C, Wang Z, Liu X, You Y, Wei H, et al. Ligustri Lucidi Fructus as a traditional Chinese medicine: a review of its phytochemistry and pharmacology. Nat Product Res. 2015;29(6):493-510.

76. Huang J, Zhang Y, Dong L, Gao Q, Yin L, Quan H, et al. Ethnopharmacology, phytochemistry, and pharmacology of Cornus officinalis Sieb. et Zucc. J Ethnopharmacol. 2017:213:280-301.
77. Yao X, Peng Y, Xu LJ, Li L, Wu QL, Xiao PG. Phytochemical and biological studies of Lycium medicinal plants. Chem Biodiver. 2011;8(6):976-1010.

78. Panossian AG. Adaptogens: tonic herbs for fatigue and stress. Altern Complement Ther. 2003;9(6):327-31.

79. Li H, Liu S-Y, Wang B. Progress of the regulation effect of ginsenosides on HPA axis. Yao xue xue bao = Acta Pharmaceutica Sinica. 2014:49(5):569-75.

80. You J, Roh KB, Li Z, Liu G, Tang J, Shin S, et al. The antiaging properties of Andrographis paniculata by activation epidermal cell stemness. Molecules. 2015;20(9):17557-69.

81. Zhu Y-P. Chinese materia medica: chemistry, pharmacology and applications. New York: CRC Press; 1998. p. 24-8.

82. Ng T. Pharmacological activity of sanchi ginseng (Panax notoginseng). J Pharm Pharmacol. 2006;58(8):1007-19.

83. Yeng C, Wai-Mei P, Alan K-WM, Choon-Keat C, Bin-Seng L, Sreenivasan $\mathrm{S}$, et al. Decreased expression of alpha-2-HS glycoprotein in the sera of rats treated with Eurycoma longifolia extract. Front Pharmacol. 2015;6(211):1-6.

84. Yang W-z HuY, W-y Wu, Ye M, Guo D-A. Saponins in the genus Panax L. (Araliaceae): a systematic review of their chemical diversity. Phytochemistry. 2014;106:7-24.

85. Wang T, Guo R, Zhou G, Zhou X, Kou Z, Sui F, et al. Traditional uses, botany, phytochemistry, pharmacology and toxicology of Panax notoginseng (Burk.) FH Chen: a review. J Ethnopharmacol. 2016;188:234-58.

86. Zabelinskaja-Mackova AA. Ginseng as panacea: its chemical composition and physiological influence.

87. Huang L-Z, Huang B-K, Ye Q, Qin L-P. Bioactivity-guided fractionation for anti-fatigue property of Acanthopanax senticosus. J Ethnopharmacol. 2011;133(1):213-9.

88. Özdemir Z, Bildziukevich U, Wimmerová M, Macůrková A, Lovecká P, Wimmer Z. Plant adaptogens: natural medicaments for 21 st century? ChemistrySelect. 2018;3(7):2196-214.

89. Costa C, Quaglio A, Di Stasi LJJ. Pfaffia paniculata (Brazilian ginseng) extract modulates Mapk and mucin pathways in intestinal inflammation. J Ethnopharmacol. 2018;213:21-5.

90. Gonzales GF. Ethnobiology and ethnopharmacology of Lepidium meyenii (Maca), a plant from the Peruvian highlands. Evid Based Complement Altern Med. 2012;2012:193496.

91. Xia C, Chen J, Deng J-L, Zhu Y-Q, Li W-Y, Jie B, et al. Novel macamides from maca (Lepidium meyenii Walpers) root and their cytotoxicity. Phytochem Lett. 2018;25:65-9.

92. Ramos MPO, Silva GDDF, Duarte LP, Peres V, Miranda RRS, Souza GHBD, et al. Antinociceptive and edematogenic activity and chemical constituents of Talinum paniculatum Willd. J Chem Pharm Res. 2010;2(6):265-74.

93. Reis LFD, Cerdeira CD, Paula BF, Silva JJD, Coelho LF, Silva MA, et al. Chemical characterization and evaluation of antibacterial, antifungal, antimycobacterial, and cytotoxic activities of Talinum paniculatum. Revista do Instituto de Medicina Tropical de São Paulo. 2015;57(5):397-405.

94. Singh N, Nath R, Lata A, Singh S, Kohli R, Bhargava K. Withania somnifera (ashwagandha), a rejuvenating herbal drug which enhances survival during stress (an adaptogen). Int J Crude Drug Res. 1982;20(1):29-35.

95. Panossian A. Understanding adaptogenic activity: specificity of the pharmacological action of adaptogens and other phytochemicals. Ann N Y Acad Sci. 2017;1401(1):49-64.

96. Maslova L, Kondrat'ev B, Maslov L, Lishmanov I. The cardioprotective and antiadrenergic activity of an extract of Rhodiola rosea in stress. Eksperimental'naia i klinicheskaia farmakologiia. 1994;57(6):61-3.

97. Spasov AA, Wikman GK, Mandrikov VB, Mironova IA, Neumoin W. A double-blind, placebo-controlled pilot study of the stimulating and adaptogenic effect of Rhodiola rosea SHR-5 extract on the fatigue of students caused by stress during an examination period with a repeated low-dose regimen. Phytomedicine. 2000;7(2):85-9.

98. Verma S, Rajeevan V, Bordia A, Jain V. Greater cardamom (Amomum subulatum Roxb.) - a cardio-adaptogen against physical stress. J Herb Med Toxicol. 2010;4(2):55-8.

99. Bhattacharya S, Muruganandam A. Adaptogenic activity of Withania somnifera: an experimental study using a rat model of chronic stress. Pharmacol Biochem Behav. 2003:75(3):547-55 